\title{
Mira kinematics in the post-Hipparcos era
}

\author{
N. Kharchenko ${ }^{1}$, E. Kilpio², O. Malkov² ${ }^{2}$ and E. Schilbach ${ }^{3}$ \\ 1 Main Astronomical Observatory, Golosiiv, Kiev - 127 03680, Ukraine \\ e-mail: nkhar@mao.kiev.ua \\ 2 Institute of Astronomy of the Russian Acad. Sci., 48 Pyatnitskaya St., Moscow 109017, Russia \\ e-mail: lena@inasan.rssi.ru; malkov@inasan.rssi.ru \\ 3 Astronomisches Rechen-Institut Heidelberg, Mönchhofstraße 12-14, 69120 Heidelberg, Germany
}

Received 11 July 2000 / Accepted 19 December 2001

\begin{abstract}
The complete data set of Mira variables from the 4th edition of the General Catalog of Variable Stars was analyzed and supplemented by the proper motions and radial velocities presently available for Miras. The resulting sample of 724 Miras with periods between 78 and 612 days contains proper motions reduced to the Hipparcos system, radial velocities and $V$ magnitudes. For each of 10 subgroups of Miras divided according to their periods and spectral types, statistical parallaxes were determined by application of five different methods. The mean absolute magnitudes, the spatial velocities and their dispersions as well as the elements of the Galactic orbits were computed as functions of the periods. The $\left(M_{\mathrm{bol}}-\log P\right)$ relation obtained was found to be considerably steeper than the PLR usually assumed for LMC Miras. For Miras in the period range 145-200 days, no significant net motion radially outwards in the Galaxy as suggested by Feast \& Whitelock (2000) was found. The predicted frequency of Miras was computed as a function of the visual magnitude.
\end{abstract}

Key words. stars: fundamental parameters - stars: variables: general

\section{Introduction}

Miras are the most numerous variables among bright pulsating stars. They are classified as red giants located at the top of the giant branch. At maximum, their absolute visual magnitude can rise above $-3^{\mathrm{m}}$ and, therefore, they can be found at large distances from the Sun. The ages of the Miras range from $10^{9}$ to $10^{10}$ years, thus they reach ages comparable to the age of the Galaxy.

Although a well-defined period-luminosity relation (PLR) has been observed for Miras in the Large Magellanic Cloud (Feast et al. 1989), there is still a problem in using these variables as universal distance indicators inside and outside the Galaxy. The main difficulty consists of the fact that according to the Mira pulsation theory, this relation is not applicable to all Long Period Variables (LPVs), but must be considered in context with the star formation history of the stellar system to which they belong. This requires a knowledge of the chemical composition and present mass distribution, i.e. on parameters that are usually poorly determined (e.g., Feast \& Whitelock 1999; Barthès \& Luri 2001). Another problem is the dependence of the PLR on the pulsation mode of Mira variables. From theoretical works (Barthès 1998),

Send offprint requests to: E. Schilbach, e-mail: elena@ari.uni-heidelberg.de the PLRs for Miras of solar mass and solar metallicity and pulsating with the same period but in different modes may yield absolute magnitude differences of more than $1^{\mathrm{m}}$. According to the analysis of available data on Mira luminosities, a majority of the Miras pulsates probably in the same mode, although other modes may be valid for a significant fraction of these variables (e.g., Feast 1999).

A reliable study of the Mira PLRs and a proof of the theory of pulsation for the Miras would require accurate data on luminosities and the linear diameters of Mira variables pulsating in different modes and within a wide range of periods. However, due to the relatively large distances of the Mira variables, their direct luminosity calibration via trigonometric parallaxes is still extremely difficult. Determining the trigonometric parallaxes of 18 Miras with a relative accuracy better than $33 \%$, Hipparcos improved the existing situation (e.g., Alvarez et al. 1997; Bedding \& Zijlstra 1998; van Leeuwen et al. 1997) but did not solve the problem which, probably, must be delayed to the future when accurate distances of numerous Mira variables will become available. At present, statistical methods provide the only possibility to estimate the absolute magnitudes of a larger number of Mira variables. Nevertheless, as long as no clear approach has been found how a mass and metallicity effect on the PLR can be taken into account and how Miras can be separated according 
to their mode of pulsation, we can derive only a general tendency of the Mira PLR which may vary depending on the proportion of stars of given properties in a considered sample.

Investigating a sample of 154 Miras and 203 Semiregulars from the Hipparcos Catalogue, Barthès et al. (1999) found that these stars could be divided into four groups differing by their kinematics. The method is based on a maximum-likelihood algorithm and the usage of Hipparcos parallaxes and proper motions as well as radial velocities, photometry, periods etc. For each of the groups, the authors also obtained the spatial distribution and the distribution of luminosity, period and color. Although Miras fall in each of the four groups, the majority $(66 \%)$ was found to belong to one group.

Recently, a series of three papers (Whitelock et al. 2000; Whitelock \& Feast 2000; Feast \& Whitelock 2000) was published dealing with Miras observed by Hipparcos. From infrared photometry and Hipparcos parallaxes, an infrared (K) PLR was obtained for Miras in the Solar neighbourhood. The Mira kinematics was investigated on the basis of Hipparcos proper motions and published radial velocities. It was found that Miras in the period range 145-200 days show a net radial motion outwards in the Galaxy.

The PLR for Mira variables in the $V$-band based on the method of statistical parallaxes was derived by Osvald \& Risley (1961), Clayton \& Feast (1969), Kharchenko (1994). In recent years, new and accurate kinematic data were published for Mira variables such as the Hipparcos (ESA 1997) and Tycho-2 (Høg et al. 2000) catalogues for proper motions and the compilation of Barbier-Brossat \& Figon (2000) for radial velocities. Therefore, a revision of the previous results by the use of new data can be a timely and useful task.

We checked the complete data set of Mira variables listed in the 4th edition of the General Catalog of Variable Stars, estimated the completeness of the catalogue with respect to Miras and supplemented the data with the proper motions and radial velocities available for the Miras at present. The results of the analysis are described in Sects. 2 and 3. Based on the final sample of 724 Miras, we determine the statistical parallaxes and mean absolute magnitudes and compare the resulting PLR with theoretical predictions in Sect. 4. We present the kinematic parameters and predictions of the frequency of Mira variables in Sect. 5. Finally, the conclusions are given in Sect. 6 .

\section{Mira-like variables in the General Catalog of Variable Stars}

\subsection{Selection procedure and reduction of stellar magnitudes to the Johnson $V$ band}

The 4th edition of the General Catalog of Variable Stars, hereafter referred to as the GCVS-4 (Kholopov et al. 1985-1998), and the New Catalogue of Suspected Variable
Table 1. Photometric reduction.

\begin{tabular}{ccr}
\hline $\begin{array}{c}\text { Photometric } \\
\text { band, } x\end{array}$ & $\begin{array}{r}(V-x) \text { color } \\
{[\mathrm{mag}]}\end{array}$ & $\begin{array}{r}\text { Number of } \\
\text { stars }\end{array}$ \\
\hline$P$ & -1.45 & 4098 \\
$B$ & -1.56 & 62 \\
$V$ & 0.00 & 545 \\
$R$ & +2.13 & 10 \\
$I$ & +4.06 & 289 \\
$J$ & +4.96 & 1 \\
$H$ & +5.90 & 1 \\
$K$ & +6.20 & 36 \\
\hline
\end{tabular}

Stars (Kukarkin et al. 1982) contain 6166 long-period variables of the Mira type. For 1110 of them, periods are missing in the catalogues, for other 14 stars no estimates of their brightness are given. Therefore, we excluded these stars from further analysis.

Photometric data in the GCVS-4 are extremely heterogeneous, and for a wide majority of the Miras only photographic magnitudes ( $P$ in Table 1$)$ are known. In order to convert the GCVS magnitudes to the Johnson $V$-band, we used in a first step the intrinsic color indices from Straižys (1992) for red giants of M5III type which corresponds to the mean spectral type of the Mira variables in the sample considered. Then, a correction $P=B-0.11$ (Allen 1973) was introduced for the stars with photographic magnitudes. The corresponding color indices are given in Table 1 together with the numbers of the GCVS stars in each subgroup. Finally, the reddening was taken into account by a statistical approach (see Chapter 4 below).

Since the magnitudes at maximum are the most complete and accurate photometric data in the catalogue, we used this indicator of brightness (hereafter marked simply as $V$ ) as the basic input parameter to obtain the results presented in the following sections.

\subsection{Completeness of the GCVS-4}

Usually, the completeness of a catalogue is defined by a magnitude where the observed frequency function $A_{\mathrm{o}}(\mathrm{m})$ of the apparent magnitudes stops growing. This approach neglects, however, selection effects and gives, as a rule, an overestimation. In order to determine the completeness of the GCVS-4 with respect to Miras, we used a method applied already by Kharchenko (1994) to find the completeness of the GCVS-3 (Kukarkin et al. 1969). The method is based on a comparison between the numbers of Miras predicted by a model and those actually found in the catalogue. We assumed the spatial distribution of Miras as being consistent with a Galactic model including a spheroid and set of disk subsystems. The frequency 

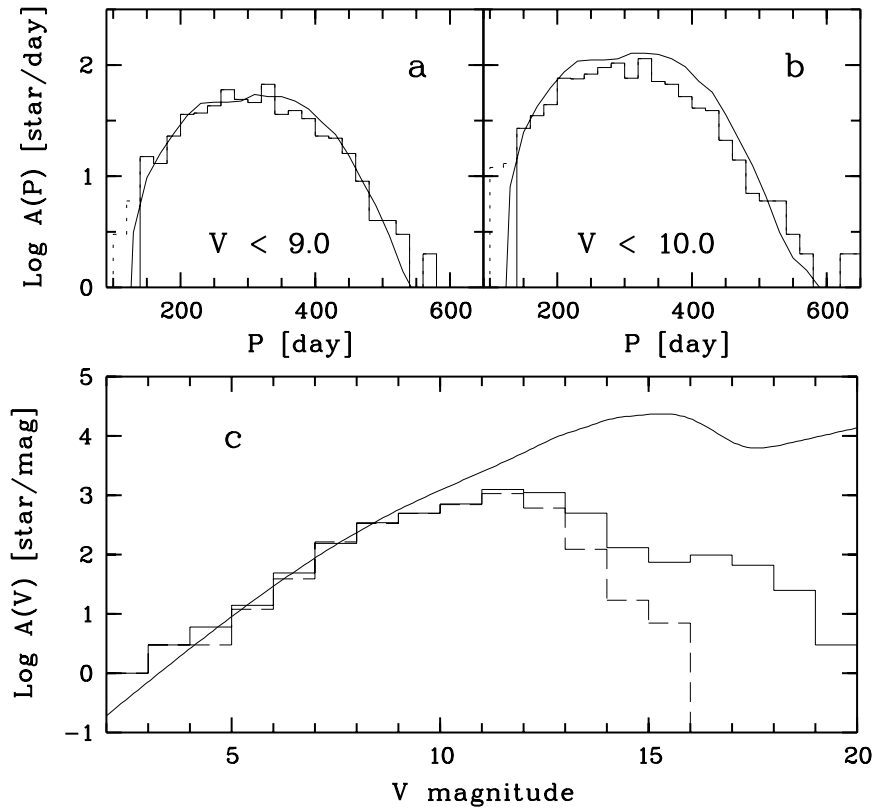

Fig. 1. Numbers of Miras predicted (curves) and listed in the GCVS-4 (histograms) plotted as a function of the period for the following two groups: Miras with magnitudes at maximum $V<9.0$ a) and $\left.V<10^{\mathrm{m}} 0 \mathbf{b}\right)$. The lower panel shows the distribution of Miras in the GCVS-4 (histogram, solid curve) and in the GCVS-3 (histogram, dashed curve) versus magnitude at maximum $V$. The solid curve indicates the predicted distribution.

function $A_{\mathrm{c}}(V)$ of the Mira variables divided into $j$ subgroups in accordance with their periods is:

$$
\begin{array}{r}
A_{\mathrm{c}}(V)=\sum_{j} \int_{-\pi / 2}^{+\pi / 2} \mathrm{~d} b \int_{0}^{2 \pi} \mathrm{d} l \int_{V_{1}}^{V_{2}} \mathrm{~d} V \int_{0}^{r_{\lim }} D_{j}\left(\boldsymbol{r}, M_{V}\right) \\
\times F_{j}\left(M_{V}\right) r^{2} \mathrm{~d} r
\end{array}
$$

where $l, b$ are galactic coordinates, $r$ is the distance from the Sun $\left(r_{\text {lim }}\right.$ ranges from 10 to $50 \mathrm{kpc}$ depending on the direction of integration $l, b), D\left(\boldsymbol{r}, M_{V}\right)$ is the density distribution proposed by Bahcall \& Soneira (1980) and $F\left(M_{V}\right)$ is the luminosity function as determined in present paper (see Chapter 5 below). A similar equation can be also written for the frequency function of the periods:

$$
\begin{array}{r}
A_{\mathrm{c}}(P)=\sum_{j} \int_{-\pi / 2}^{+\pi / 2} \mathrm{~d} b \int_{0}^{2 \pi} \mathrm{d} l \int_{V_{1}}^{V_{2}} \mathrm{~d} V \int_{0}^{r_{\lim }} D_{j}\left(\boldsymbol{r}, P\left(M_{V}\right)\right) \\
\times \Phi_{j}\left(P\left(M_{V}\right)\right) r^{2} \mathrm{~d} r .
\end{array}
$$

The results of the comparison are shown in Fig. 1. Even if the GCVS- 4 contains about 2000 Miras more than the GCVS-3, we must conclude that both catalogues are complete up to magnitude at maximum $V=9{ }^{\mathrm{m}} 0$. According to Figs. 1 and 2, the Miras added in the GCVS-4 are mostly faint stars located in a few sky areas.

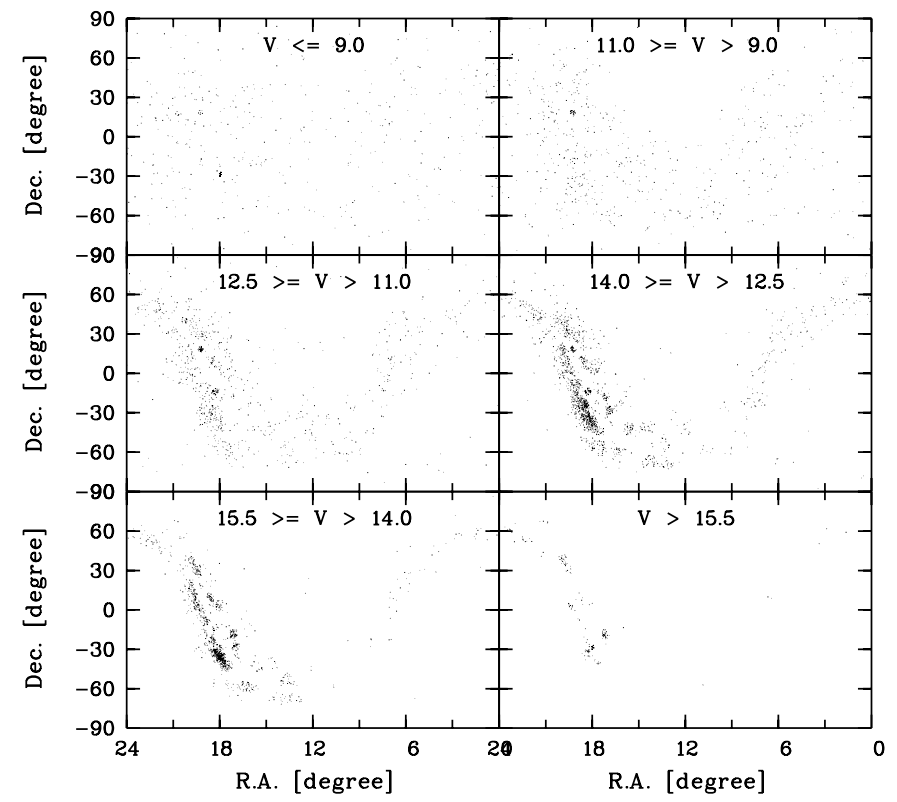

Fig. 2. Distribution of Mira variables from the GCVS-4 on the sky (in equatorial coordinates) for different magnitude ranges.

Table 2. Sources for the proper motion data.

\begin{tabular}{lc}
\hline Catalogue & Number of Miras \\
\hline Hipparcos & 239 \\
Tycho-2 & 780 \\
Tycho-1 & 452 \\
ACT RC & 534 \\
TRC & 382 \\
CMC11 & 323 \\
PPM & 247 \\
Kharchenko (1992) & 303 \\
\hline
\end{tabular}

\section{The compiled catalogue of kinematic characteristics of the Miras}

\subsection{Proper motions}

The proper motion data for the Mira variables are relatively rare and rather heterogeneous. Analysing the data available before the end of eighties, Kharchenko (1992) reduced proper motions of 303 Miras to a common reference system. During the last decade, a number of new catalogues containing proper motions of Mira variables was published. These catalogues are: PPM (Röser \& Bastian 1991; Bastian \& Röser 1993a,b), Hipparcos and Tycho-1 (ESA 1997), ACT RC (Urban et al. 1998), TRC (Høg et al. 1998), CMC11 (1999) and Tycho-2 (Høg et al. 2000). The numbers of Miras in the catalogues are listed in Table 2.

The proper motions from these catalogues were reduced to the Hipparcos reference system which gives, at present, the best realization of an inertial system in the optical. Using stars in common, we compared the Hipparcos proper motions $\mu_{x}^{\mathrm{H}}, \mu_{y}^{\mathrm{H}}$ with the corresponding data $\mu_{x}$, 


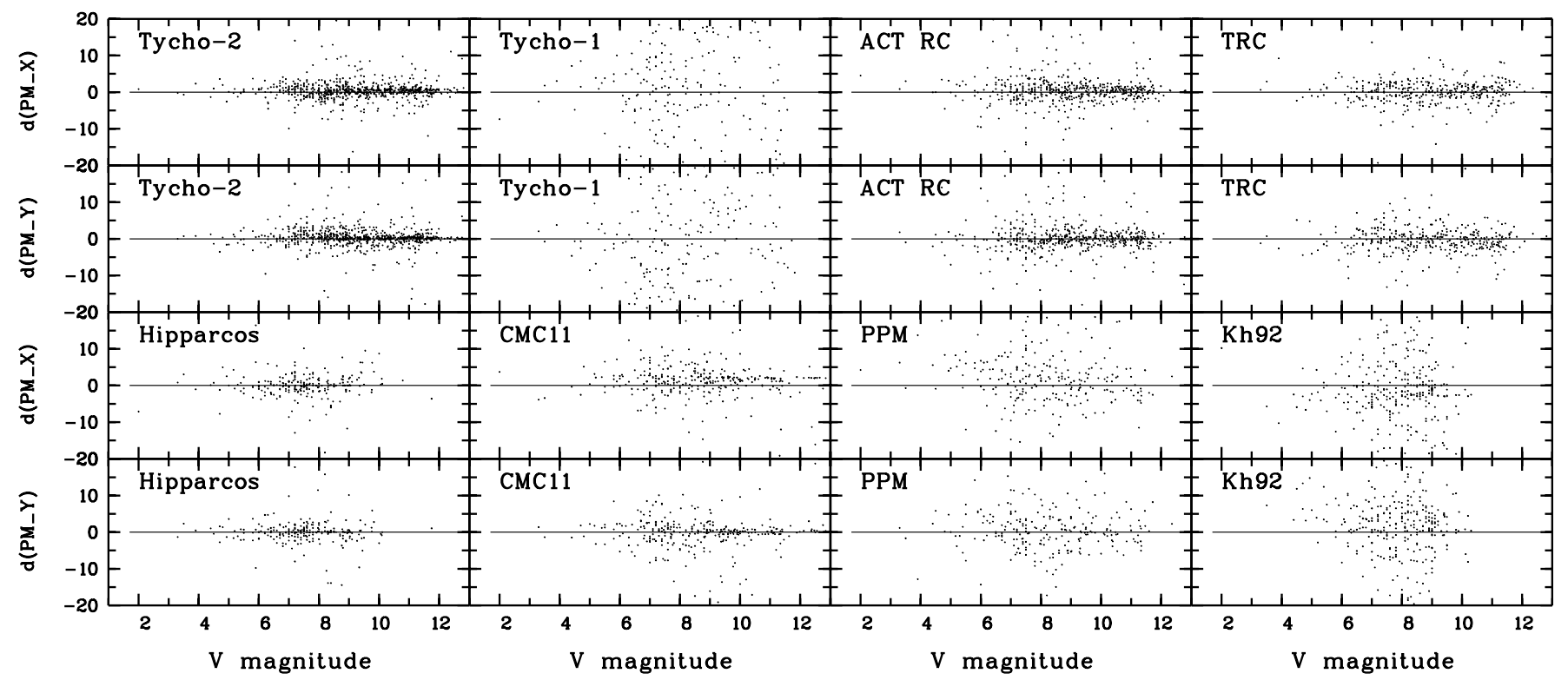

Fig. 3. Proper motion differences in mas/year between the compiled catalogue and original astrometric catalogues as a function of magnitude.

$\mu_{y}$ in each catalogue and derived corrections for different zero points $\delta \mu$ and precession terms $\Delta k, \Delta n$ as

$\mu_{x}-\mu_{x}^{\mathrm{H}}=\Delta k \cos \delta+\Delta n \sin \alpha \sin \delta+\delta \mu_{x}$

$\mu_{y}-\mu_{y}^{\mathrm{H}}=\Delta n \cos \alpha+\delta \mu_{y}$.

Finally, the mean weighted proper motions and the corresponding rms errors were computed for each star. The weight for a star in each catalogue was set in accordance with the individual proper motion error given for this star in the original catalogue. Figure 3 shows the proper motion differences between the final catalogue and the original catalogues. In total, for 1041 Mira variables, proper motions in the Hipparcos system were determined.

\subsection{Radial velocities of Miras}

We checked various catalogues and lists containing radial velocities. In nine of them the relevant data for Mira variables were found.

It is a well-known fact that the radial velocity $V_{\mathrm{r}}$ can be derived from absorption and/or from emission lines. In the case of Mira variables, the essential information on radial velocity, i.e. on the radial component of the space velocity, is obtained from absorption lines: they refer to the atmosphere of a given star and so describe its true radial velocity. On the contrary, emission lines reflect the state of star's envelope and include an additional velocity component resulting from stellar winds. Therefore, we introduced a correction in $V_{\mathrm{r}}$ computed from emission lines making use of the relation (Ikaunieks 1971):

$V_{\mathrm{r}}^{\mathrm{abs}}-V_{\mathrm{r}}^{\mathrm{em}}=0.035 P$,

where $P$ is the period.

For stars included in more than one catalogue, the $V_{\mathrm{r}}$ values were averaged. The resulting list contains $V_{\mathrm{r}}$ for
Table 3. Sources for the radial velocity data.

\begin{tabular}{lr}
\hline Reference & Number of Miras \\
\hline Feast (1963) & 403 \\
Smak \& Preston (1965) & 271 \\
Perry \& Bidelman (1965) & 11 \\
Feast (1966) & 52 \\
Feast et al. (1972) & 3 \\
Dean (1976) & 19 \\
Barnes \& Fekel (1977) & 2 \\
Feast et al. (1980) & 19 \\
Barbier-Brossat \& Figon (2000) & 58 \\
\hline
\end{tabular}

786 stars. Table 3 gives the references for the data on radial velocities and numbers of Mira variables considered in each catalogue.

The typical accuracy of a radial velocity of a Mira variable is estimated to be a few kilometers per second. The main uncertainties contributing to the resulting error are: the error of measurements, the error due to the phase and/or intrinsic variations, and the error due to dispersion in the relation between velocities from emission lines and radial velocities (Eq. (5)). For example, in their determinations of radial velocities, Smak \& Preston (1965) estimated these errors as $\pm(1-2) \mathrm{km} \mathrm{s}^{-1}, \pm(2-3) \mathrm{km} \mathrm{s}^{-1}$, and $\pm(3-4) \mathrm{km} \mathrm{s}^{-1}$, respectively. In the following, we assumed the mean error of radial velocity obtained from a single spectrogram of an average quality to be $\pm 5 \mathrm{~km} \mathrm{~s}^{-1}$.

\subsection{Sample of Mira variables}

In total, our list contains 1103 Mira variables with proper motions in the Hipparcos system and/or radial velocities. For 724 of them both proper motions and radial velocities 


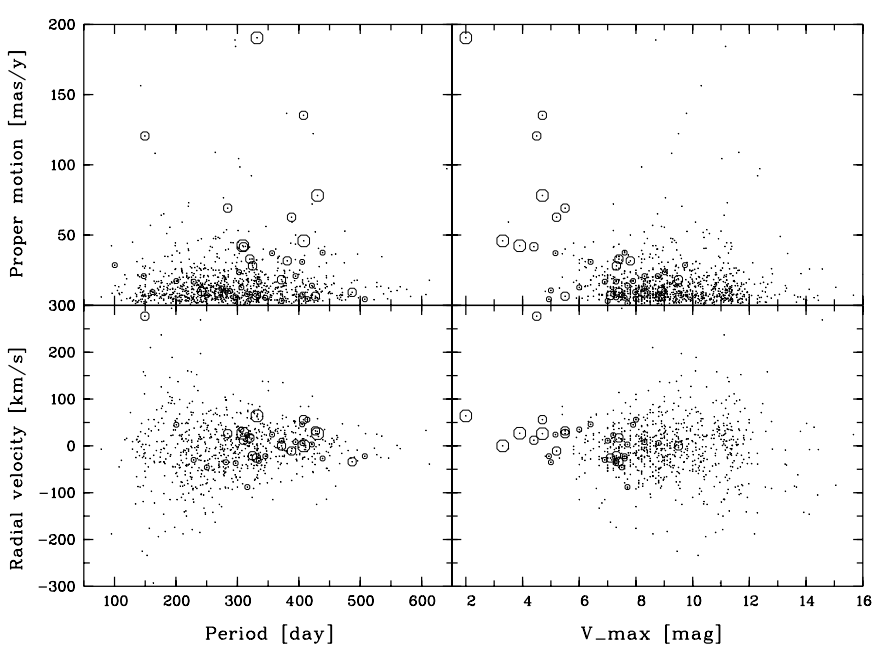

Fig. 4. Distribution of proper motions and radial velocities of Mira variables in our sample versus $V$-magnitude and period. The small, medium and large circles indicate 43 Miras whose trigonometric parallaxes were determined by Hipparcos with a relative error better than $50 \%, 33 \%$ and $15 \%$, respectively.

are available. According to the GCVS-4, 753 Miras in our list are stars of spectral types $K$ or $M, 53$ of type $C, R$ or $N$ and 41 of $S$. These Miras were classified as titanium or oxygen-rich $(\mathrm{M})$, carbon $(\mathrm{C})$ and zirconium $(\mathrm{S})$ stars, respectively. For the remaining 256 Miras, the spectral type is not known and so we assume them to be oxygenrich stars.

Figure 4 shows the distribution of radial velocities and proper motions $\left(p m=\sqrt{\left(\mu_{\alpha} \cos \delta\right)^{2}+\mu_{\delta}^{2}}\right)$ of the Miras from our sample versus $V$-magnitudes and period.

For the majority of the Mira variables, equatorial coordinates were taken from the astrometric catalogues listed in Table 2 in accordance with their priority: Hipparcos (239 stars), Tycho-2 (623 stars), Tycho-1 (38 stars), CMC11 (90 stars), PPM (8 stars). The coordinates of the remaining 105 stars were taken from the GCVS- 4 and transformed to the equinox 2000.0.

Figure 5 shows that our kinematic sample represents the complete period range covered by the GCVS-4. Its distribution over the period is consistent with the distribution of Miras brighter than $V=12$ which is the limiting magnitude of the kinematic sample. The mean and median periods of the kinematic sample agree sufficiently with those of the whole GCVS-4 sample.

\section{Distance and luminosity scale}

At relatively small distances from the Sun, there are only a few Miras known. Therefore, Hipparcos could achieve rather moderate accuracy in trigonometric parallaxes for this type of star: from 239 Miras observed by Hipparcos, only 43, 18 and 4 Miras have parallaxes with a relative accuracy better than 50\%, 33\% and 15\%, respectively. According to Fig. 4, these stars do not cover the complete range of periods and represent poorly the kinematic properties of Miras, as well.

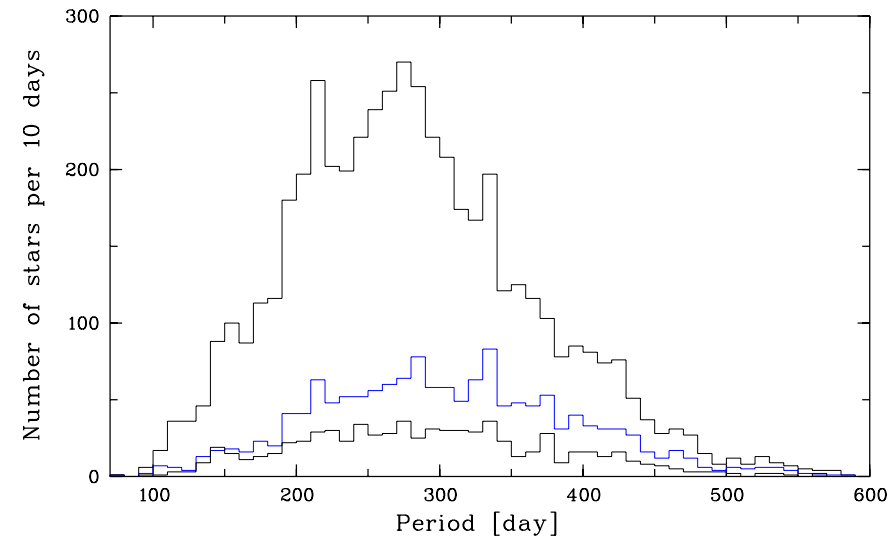

Fig. 5. Distribution of Mira variables versus period. The lines from top to bottom correspond to: all Miras listed in the GCVS-4, Miras with magnitudes at maximum $V<12$ and 724 Miras with both proper motions and radial velocities.

In this paper we applied the method of statistical parallaxes to estimate the average distances and hence the mean absolute magnitudes of Miras selected within a given range of periods and visual magnitudes. The method is based on the assumption that the peculiar motions of stars in such a group are randomly distributed, and their kinematic data, on average, reflect the solar motion. For 724 Mira variables in our sample, the proper motions in the Hipparcos system and radial velocities are available. According to their period distribution, the oxygen-rich Miras were divided into 8 groups (see Table 4).

For each group, the statistical parallax method works correctly if a sample includes a sufficient number of randomly moving stars distributed homogeneously all over the sky and chosen within a small range of magnitudes (and, consequently, distances). In order to satisfy these conditions sufficiently well, we first determined statistical parallaxes for 388 Miras selected from the basic sample in a range of magnitudes at maximum $V=7^{\mathrm{m}} \cdot 0-9{ }^{\mathrm{m}} 5$. Following the $3 \sigma$ criterion, we excluded 6 stars due to the relatively large proper motions and/or radial velocities.

For each star we computed proper motion components $\mu_{v}, \mu_{\tau}$ assuming the galactic coordinates of the solar apex to be $(l, b)=\left(27.6^{\circ} \pm 6.2^{\circ},+32.4^{\circ} \pm 1.7^{\circ}\right)$ which corresponds to the solar velocity with respect to the LSR derived by Dehnen \& Binney (1998) from the Hipparcos data (see also Chapter 5 ). The solar velocity $V_{\odot}$ relative to the Miras was derived from the radial velocities for each group, separately. All velocity components were corrected for Galactic rotation with $A=+14.82 \pm 0.84, B=$ $-12.37 \pm 0.64 \mathrm{~km} \mathrm{~s}^{-1} / \mathrm{kpc}$ (Feast \& Whitelock 1997). These values are based on the Hipparcos proper motions and, therefore, could be considered as the most reliable estimates of the Oort constants. Since in each group, the distribution of Miras over the sky is sufficiently uniform, uncertainties in the values of the Oort constants should not affect considerably the resulting statistical parallaxes. Indeed, changing the values of the Oort constants within reasonable limits we did not find any significant change in 
Table 4. Mean parallaxes, amplitudes and absolute magnitudes of Miras. The table refers to Miras with magnitudes at maximum $V$ between $7^{\mathrm{m}} 0$ and 9.5 .

\begin{tabular}{lcccccccccc}
\hline Type & $M$ & $M$ & $M$ & $M$ & $M$ & $M$ & $M$ & $M$ & $C$ & $S$ \\
Period range & $<165$ & $165-225$ & $225-270$ & $270-315$ & $315-350$ & $350-385$ & $385-435$ & $>435$ & & \\
\hline $\bar{P}$ [day] & 135 & 200 & 249 & 289 & 332 & 370 & 409 & 482 & 400 & 371 \\
No. of stars & 22 & 53 & 64 & 62 & 64 & 25 & 26 & 14 & 32 & 20 \\
$\bar{\pi}$ [mas] & 0.70 & 0.80 & 1.17 & 1.14 & 1.15 & 1.49 & 1.49 & 1.97 & 1.38 & 1.62 \\
& \pm 0.30 & \pm 0.17 & \pm 0.25 & \pm 0.25 & \pm 0.21 & \pm 0.51 & \pm 0.42 & \pm 0.78 & \pm 0.47 & \pm 0.48 \\
$\overline{M_{V}}[\mathrm{mag}]$ & -3.37 & -2.65 & -1.75 & -1.78 & -1.78 & -1.22 & -1.34 & -0.62 & -1.70 & -1.62 \\
& \pm 0.77 & \pm 0.41 & \pm 0.41 & \pm 0.43 & \pm 0.36 & \pm 0.64 & \pm 0.54 & \pm 0.72 & \pm 0.64 & \pm 0.56 \\
$\bar{A}[\mathrm{mag}]$ & 4.26 & 4.87 & 5.06 & 5.32 & 5.60 & 5.77 & 6.14 & 5.83 & 4.69 & 6.38 \\
& \pm 0.19 & \pm 0.15 & \pm 0.15 & \pm 0.15 & \pm 0.18 & \pm 0.31 & \pm 0.27 & \pm 0.38 & \pm 0.20 & \pm 0.39 \\
\hline$\overline{M_{\mathrm{bol}}}[\mathrm{mag}]$ & -2.72 & -3.61 & -3.70 & -4.33 & -4.87 & -4.75 & -5.18 & -5.42 & & \\
& \pm 0.85 & \pm 0.53 & \pm 0.53 & \pm 0.55 & \pm 0.50 & \pm 0.77 & \pm 0.67 & \pm 0.87 & & \\
\hline
\end{tabular}

the results (even in a case of $A=B=0$ the corresponding statistical parallaxes differ less than $11 \%$ from the values computed with the Oort constants of Feast \& Whitelock 1997).

For each group, the statistical parallaxes were computed in five different approaches:

- the classical upsilon component method based on the relation between the proper motion component $\mu_{v}$ and the solar velocity $V_{\odot}$

$\bar{\pi}_{v}=\left(4.74 \sum_{i}\left(\mu_{v} \sin \lambda\right)\right):\left(V_{\odot} \sum_{i}\left(\sin ^{2} \lambda\right)\right)$

where $\lambda$ is the angular distance between a star and the standard solar apex;

- the classical tau component method based on the relation between the proper motion components $\mu_{\tau}$ and the radial velocities $V_{\mathrm{r}}$

$\bar{\pi}_{\tau}=\left(4.74 \sum_{i}\left|\mu_{\tau}\right|\right): \sum_{i}\left|V_{\mathrm{r}}^{\prime}\right|$

where $V_{\mathrm{r}}^{\prime}$ is the radial velocity corrected for the solar motion;

- the relation between the solar motion with respect to the stars in a given group, computed from proper motions and radial velocities. This approach does not use any preliminary assumption on apex coordinates

$\bar{\pi}_{v, \tau}=\frac{4.74\left(u_{\odot}^{2}+v_{\odot}^{2}+w_{\odot}^{2}\right)^{0.5}}{V_{\odot}}$

where the solar motion components $u_{\odot}, v_{\odot}, w_{\odot}$ were derived from

$\mu_{x \mathrm{c}}=u_{\odot} \sin \alpha-v_{\odot} \cos \alpha$

$\mu_{y c}=u_{\odot} \cos \alpha \sin \delta+v_{\odot} \sin \alpha \sin \delta-w_{\odot} \cos \delta$.

Here $\mu_{x c}$ and $\mu_{y c}$ are the proper motion components corrected for the Galactic rotation;
- the relation between the upsilon component dispersion and radial velocity dispersion

$\bar{\pi}_{v, V_{\mathrm{r}}}=4.74 \frac{\sigma_{\mu_{v}}}{\sigma_{V_{\mathrm{r}}}}$

- the relation between the tau component dispersion and radial velocity dispersion

$\bar{\pi}_{\tau, V_{\mathrm{r}}}=4.74 \frac{\sigma_{\mu_{\tau}}}{\sigma_{V_{\mathrm{r}}}}$

For each group and each method, the rms errors of the results were estimated from the propagation of the mean errors of the input data. In each case, the scatter of parallaxes was obtained to be less than 2 mas.

The statistical parallaxes and their rms errors computed for each group by the different methods do not differ considerably and thus were averaged. The results are given in Table 4.

Based on the statistical parallaxes, the corresponding mean absolute magnitudes at maximum were derived for each group by iteration of the equations:

$M_{V}=V-5 \log r+5-A_{V}(r, b)$

$A_{V}(r, b)=a_{V} h_{\mathrm{Za}}\left[1-\exp \left(-r|\sin b| / h_{\mathrm{Za}}\right)\right] /|\sin b|$

where $b$ is the galactic latitude and $A_{V}(r, b)$ is the total interstellar extinction at a distance $r$ from the Sun. The corresponding reddening is $E_{B-V}=A_{V} / 3.1$. After a critical analysis of different interstellar extinction models (Kilpio \& Malkov 1997), the extinction in the Galactic plane per $1 \mathrm{kpc}, a_{V}$, and the scale height of the extinction layer, $h_{\mathrm{Za}}$, were taken from Sharov (1963) where they are given in dependence of the direction and the limiting distance. For larger distances, the mean values $\overline{a_{V}}=1.6 / \mathrm{kpc}$ and $\overline{h_{\mathrm{Za}}}=114 \mathrm{pc}$ were assumed.

In general, from 5 to 10 iterations were needed to obtain convergent solutions of (13) and (14) for different groups. The corresponding PLR based on 330 oxygen-rich Miras with magnitudes at maximum $V$ between 7.0 and 


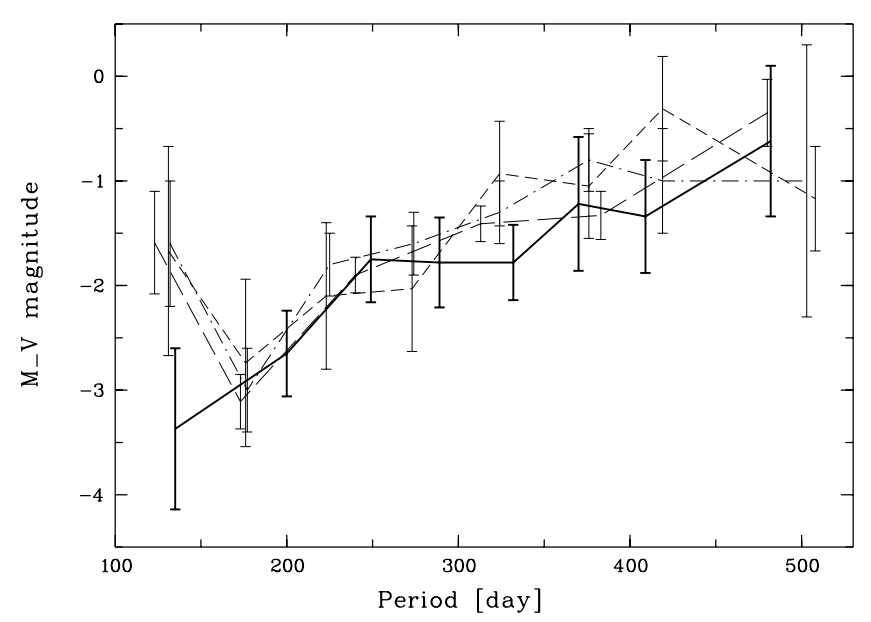

Fig. 6. Absolute magnitude versus period for the oxygenrich Miras (solid curve). For comparison, the previous results obtained for Mira variables are presented. Short-dashed curve: Osvald et al. (1961), dashed-dotted curve: Clayton et al. (1969), long-dashed curve: Kharchenko (1992).

9.5 is presented in Fig. 6 and Table 4. The errors given for $\overline{M_{V}}$ are the rms errors of the mean absolute magnitudes at maximum obtained for each group. We have to emphasize that two important factors, uncertainties in the total interstellar extinction $A_{V}(r, b)$ and in the apparent magnitudes at maximum $V$, are not explicitly included in the error estimation of absolute magnitudes since, generally speaking, they are not known for each Mira. By the determination of the mean absolute magnitude from a large sample of homogeneous and uniformly distributed stars, we can expect that, to a certain extent, these factors compensate for themselves. On the other hand, errors in $A_{V}(r, b)$ and $V$ (among other uncertainties) fully contribute to a scatter of estimated individual absolute magnitudes and, consequently, to the rms errors of the mean absolute magnitudes in Fig. 6 and Table 4.

In the next step we took this PLR to derive the absolute magnitudes at maximum $M_{V}$ for the remaining stars of the kinematic sample $(724-382=342$ stars $)$. The distances $r$ and extinctions $A_{V}(r, b)$ were computed from (13) and (14).

\subsection{Comparison with Hipparcos parallaxes}

Due to large amplitudes of Mira variables, Hipparcos could not observe the complete light curves for a number of these stars. Especially for those with periods close to 1 year, the parallax determination was rather difficult. We could find only 11 resolved stars with a relative accuracy of the parallaxes better than 25\% from 239 Miras observed by Hipparcos. Nevertheless, these data are the best that are available for Miras at the moment. The result of a comparison between the Hipparcos parallaxes and parallaxes obtained from the PLR as described in the previous paragraph is shown in Fig. 7 . The error bars for $\pi_{\mathrm{PLR}}$ indicate the rms errors estimated by the propagation of the mean errors listed in Table 4 for a given group. Except for Mira

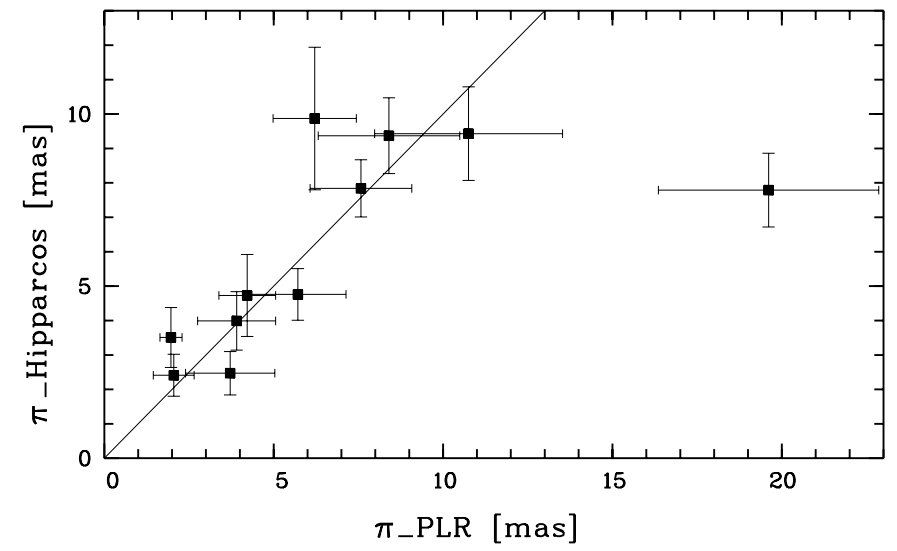

Fig. 7. Comparison of the Hipparcos parallaxes with parallaxes derived in this paper from the PLR. The solid line indicates zero parallax differences.

itself (the point most on the right in Fig. 7), the coincidence is quite good.

The "outlier behaviour" of Mira may be a consequence of a strong variability of its $V$ magnitude at maximum. Though Mira becomes occasionally as bright as $V=2^{\mathrm{m}}$ (GCVS-4), its average magnitude at maximum is about 3. 4 (GCVS-3). In the first case, the statistical method yields a parallax $\pi_{\mathrm{PLR}}=19.6$ mas (Fig. 7 ), in the second case $\pi_{\mathrm{PLR}}=9.8$ mas, which is in a better agreement with the Hipparcos parallax (7.8 mas). On the other hand, due to the binarity (=ADS 1778) and the pulsation period close to 1 year ( $\approx 332$ days), Mira was not an "easy" object for Hipparcos, too. This is indicated by large correlation coefficients (up to $39 \%$ ) between the astrometric parameters of Mira in the Hipparcos catalogue. Note also that Mira was not used in the construction of the PLR which, in this paper, was based on stars with $V$ magnitudes at maximum between 7.0 and 9.5.

\subsection{Period - bolometric luminosity relation}

In order to compare the period-luminosity relation derived in this paper with theoretical predictions of the pulsation theory and with the relation for LMC Miras from Feast \& Whitelock (1999), we must convert the averaged visual magnitudes at maximum brightness into mean bolometric magnitudes.

Since visual amplitudes of Miras tend to correlate with periods (Whitelock et al. 2000), we computed the averaged amplitudes for each group, separately. We used all stars from our basic kinematic sample (724 Miras) with amplitudes listed in the GCVS-4. The mean amplitudes $(\bar{A})$ and their rms errors are given in Table 4. For each group, the mean absolute visual magnitudes were derived as $\overline{M_{V}}=\overline{M_{V}}+0.5 \bar{A}$.

Figure 8 shows a BC-period relation for the Mira variables having reliable Hipparcos data. Periods, Hipparcos parallaxes, extinction data and bolometric (the average of the maximum and minimum) magnitudes were taken from van Leeuwen et al. (1997); visual magnitudes are averaged GCVS-4 data. 


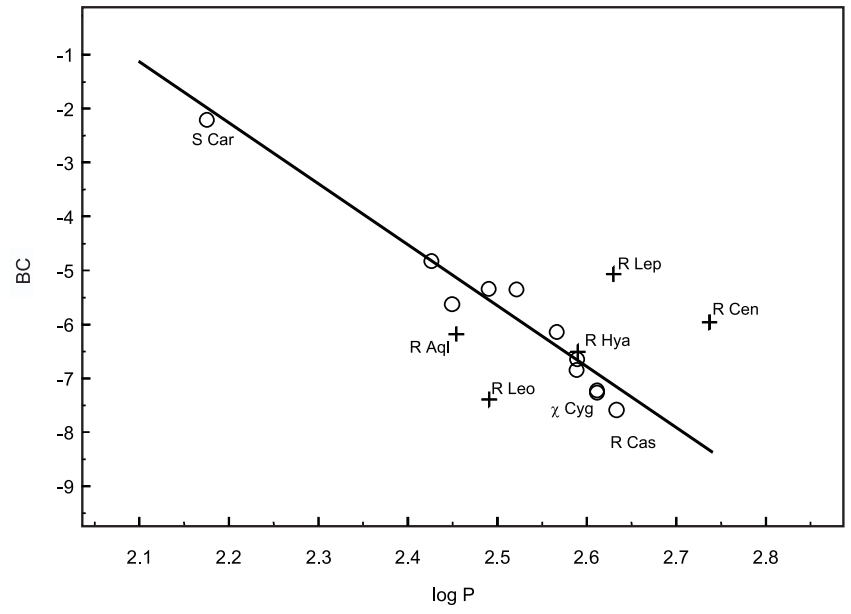

Fig. 8. BC - period relation for the Mira variables (open circles). The stars excluded from further analysis are marked with crosses.

The following Miras listed in van Leeuwen et al. (1997) were excluded from further analysis: the carbon-rich $\mathrm{R}$ Lep, the double-period $\mathrm{R}$ Cen, two stars with decreasing periods (R Aql and R Hya) and R Leo (its ground-based trigonometric parallax differs from the Hipparcos result). These five stars are marked with crosses. For the remaining 11 Miras (indicated by circles), a linear fit to the data yields

$B C=-11.3( \pm 0.8) \times \log P+22.6( \pm 2.1), \quad \chi^{2}=1.11$.

Since the distribution of stars over the pulsation period is rather inhomogeneous, we checked the possibility whether the shape and slope of the obtained relation is mainly determined by S Car, the shortest-period star in our sample. A least squares solution without $\mathrm{S}$ Car yields

$B C=-12.4( \pm 1.6) \times \log P+25.3( \pm 4.2), \quad \chi^{2}=1.03$.

Since the coefficients in (15) and (16) did not differ significantly, we conclude that the obtained solution is sufficiently stable.

We may also assume that the derived relation is almost independent of the mode of pulsation: $\chi \mathrm{Cyg}$ and $\mathrm{R}$ Cas identified by van Leeuwen et al. (1997) as fundamental pulsators match the relation as well as other stars, presumably first overtone pulsators. We note, however, that a reliable interpretation of the pulsation mode of Miras is still a controversial and highly discussed problem (e.g., see discussion in Feast 1999; Tuchman 1999; Percy \& Bagry 1999).

The corresponding BCs were applied to the mean visual magnitudes (see Table 4) computed for eight groups of oxygen-rich Mira variables. The resulting $\left(M_{\mathrm{bol}}-\log P\right)$ relation is presented in Fig. 9 and in the bottom line of Table 4 .

\subsection{Confrontation with theoretical and empirical $M_{\text {bol }}-\log P$ relations}

The results were compared with theoretical predictions of Barthès (1998) for near-solar metallicity $(Z=0.02)$.

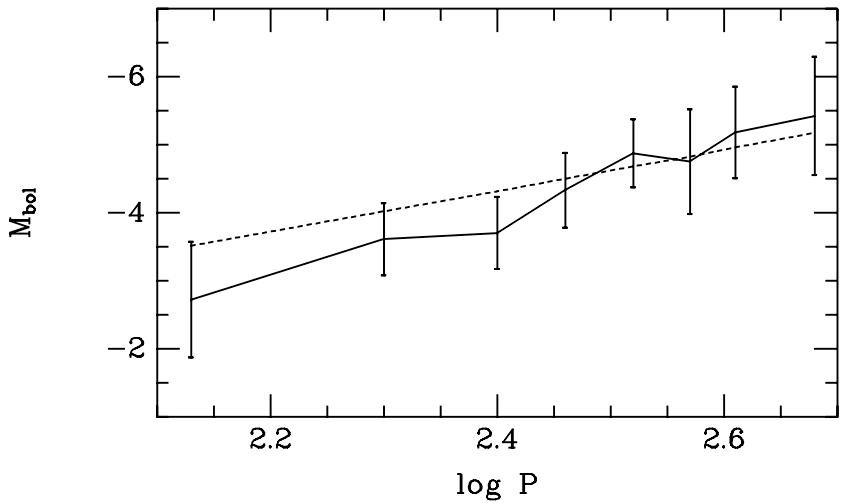

Fig. 9. The $\left(M_{\mathrm{bol}}-\log P\right)$ relation. Solid and dashed lines represent our results and data from van Leeuwen et al. (1997), respectively.

According to the model grid by Barthès (1998), our $\left(M_{\mathrm{bol}}-\log P\right)$ relation is compatible with both fundamental mode and first overtone pulsations. Nevertheless, Miras with shorter periods $(P<270$ days $)$ fit better the fundamental mode with masses lower than $1.5 M_{\odot}$, whereas Miras with larger periods pulsate probably on the first overtone with masses between $0.8 M_{\odot}$ and $2 M_{\odot}$. The mid-point of our sample at $P=285$ days matches the first overtone with a mass of about $1 M_{\odot}$ or alternatively, the fundamental mode with a mass of about $2 M_{\odot}$. The model of Barthès (1998) is based on a linear pulsation model assuming that the pulsating envelope does not significantly differ from the static one. However, further developments (Ya'ari \& Tuchman 1996; Barthes \& Luri 2001) have provided indication that this assumption may not be correct.

Recently, theoretical $\left(M_{\mathrm{bol}}-\log P\right.$ ) relations based on an improved linear nonadiabatic pulsation model have been computed by Barthès \& Luri (2001) for different masses and pulsation modes but only for a LMC metallicity $(Z=0.008)$. We have compared our data with this model grid keeping in mind that a smaller metallicity leads to a higher luminosity; e.g. metallicity decreasing by a factor of 20 leads to an increase of about $0.43 \mathrm{mag}$ in luminosity (Barthès 1998). On the other hand, a metallicity of $Z=0.008$ may be a good compromise for Miras in the solar neighbourhood provided that these stars belong to an older population. From the comparison with the model grid by Barthès \& Luri (2001) for LMC metallicity, we can conclude that our $\left(M_{\mathrm{bol}}-\log P\right)$ relation is located in the region occupied by Miras pulsating in the fundamental mode with masses from $0.6 M_{\odot}$ (shorter periods) and up to $2 M_{\odot}$ (larger periods). Though, taking into account the error bars of $M_{\mathrm{bol}}$, Miras with periods larger than 315 days in our sample are also compatible with the first overtone and masses of about $1 M_{\odot}$. The mid-point of the sample fits the fundamental mode with about $1 M_{\odot}$ mass. Due to relatively large rms errors of the bolometric magnitudes (see Table 4), this interpretation will probably be valid also if we could compare our results with model predictions for near-solar metallicity. The nearly identical conclusions can be drawn from comparing our results with 
the models by Wood \& Sebo (1996) for the asymptotic giant branch where $Z=0.008$ was adopted, too.

To compare our empirical $\left(M_{\mathrm{bol}}-\log P\right)$ relation with other observations, we plotted the PLR by van Leeuwen et al. (1997) in Fig. 9. This relation adopts the slope derived for LMC Miras (Feast et al. 1989) and the zero-point evaluated from the Hipparcos data. The corresponding infrared (K) PLR were used by Feast \& Whitelock (2000) to study the kinematics of Miras measured by Hipparcos (see discussion in Chapter 5). Although the differences between the PLRs in Fig. 9 are within error bars, our PLR is significantly steeper than the PLR by van Leeuwen et al. (1997).

Several reasons for these differences may be considered. Relatively large uncertainties in the Hipparcos parallaxes of Miras (see, e.g., discussion in Feast \& Whitelock 1999) can introduce a systematic effect on the derived $\left(M_{\mathrm{bol}}-\log P\right)$ relation. Also, the empirical amplitudeperiod relation used can yield a bias in the calculation of mean absolute magnitudes, though the number of stars is relatively large (from 23 in the last group to 112 in the third group). To check this, we re-calculated the amplitude-period relation for a subsample of bright stars $(7.0 \leq V \leq 9.5)$ and obtained the same results.

On the other hand, we have to recall that the considered sample of Miras is by far not homogeneous and may include stars of different populations and in a wide range of metallicities and masses. It may also be a mixture of fundamental mode pulsators, first overtone pulsators and, probably, higher overtone pulsators. Due to a different evolution history, Miras in our Galaxy and in the LMC must not necessary show the same PLR. A similar conclusion was inferred by Barthès \& Luri (1999) who also derived a considerably steeper $\left(M_{K}-\log P\right)$ relation of their Group 1 of galactic oxygen-reach LPVs (composed to about $66 \%$ of Miras) than usually observed in the LMC. The steepness of the PLR in the solar neighbourhood can result from a present mass distribution which may differ from the mass distribution of Miras in the LMC. Additionally, an apparent bend of the PLR at $\log P \approx 2.35$ (Fig. 9) may indicate distinct populations of Miras in the solar neighbourhood. This suggestion is also supported by the kinematics of the Mira sample studied (see Chapter 5). On the other hand, a more or less continuous star formation may be characteristic of the LMC (see also Barthès \& Luri 1999). However, at present, there is no reliable observing basis for a conclusive answer.

\section{Kinematic parameters and frequency of Mira variables}

\subsection{Mira kinematics}

We used the distances, proper motions and radial velocities of the Miras to compute the spatial velocity components $U, V, W$ and their dispersions in the Galactic Cartesian system with the axes directed to the anticenter, along the rotation, and to the north pole of the Galaxy, respectively. The spatial velocities were corrected for the peculiar velocity of the Sun assuming $(U, V, W)_{\odot}=(-10.0 \pm 0.4,+5.2 \pm 0.6,+7.2 \pm 0.4) \mathrm{km} \mathrm{s}^{-1}$ (Dehnen \& Binney 1998). The elements of Galactic box orbits, apocenter and pericenter distances $R_{\mathrm{a}}$ and $R_{\mathrm{p}}$, eccentricity $e$ and maximum $Z$-distance from the Galactic plane $Z_{\max }$ which a star can reach in its orbital motion, were obtained by assuming the form of the Galactic potential proposed by Saio \& Yoshii (1979). The distance of the Sun from the Galactic center is taken to be $8.5 \mathrm{kpc}$. According to the $3 \sigma$-criterion, 69 stars were excluded from the final solution. The resulting values and their rms errors are presented in Table 5 .

The kinematic data given in Table 5 indicate a higher age for the oxygen-rich Miras with smaller periods. Smaller $\bar{V}$-velocities and larger velocity dispersions $\overline{\sigma_{U}}, \overline{\sigma_{V}}, \overline{\sigma_{W}}$ support the idea that these stars belong to older Galactic subsystems. Also, the increasing eccentricities and maximum $Z$-distances of the oxygen-rich Miras with smaller periods prove that these stars move at higher eccentric orbits, more inclined to the Galactic plane, that is typical for the older population.

This conclusion is consistent with the results obtained by Barthès et al. (1999) for oxygen-rich LPVs falling into their Groups 1 and 4 which are predominantly composed of Miras (102 Miras out of 121 stars in Group 1 and 13 Miras out of 14 stars in Group 4). A direct comparison of the kinematic parameters is rather difficult since LPVs in these Groups cover larger ranges of periods $P$ (from 225 to 500 days with $\bar{P} \approx 300$ days in Group 1 and from 125 to 250 days with $\bar{P} \approx 170$ days in Group 4). However, the velocity components derived by Barthès et al. (1999) for Groups 1 and 4 coincide well with the corresponding values in Table 5 for Miras with periods $P=270 \div 315$ days and $P<165$ days, respectively.

Recently, a kinematic study of field Miras with proper motions measured by Hipparcos was published by Feast $\&$ Whitelock (2000). For the determination of space velocities, the authors make use of the infrared (K) PLR for LMC Miras (Feast et al. 1989) with the zero-point derived from Hipparcos parallaxes (Whitelock \& Feast 2000). The kinematic parameters obtained by the authors are in good agreement with our results for Miras with periods lager than 200 days $^{1}$. For variables with periods between 145 and 200 days (17 stars), a significant motion radially outwards from the Galactic Centre of $75 \pm 18 \mathrm{~km} \mathrm{~s}^{-1}$ was derived in Feast \& Whitelock (2000).

In our sample there are 77 stars with periods in the range 145-200 days. Out of them, 11 are in common with stars listed by Feast \& Whitelock (2000) in their group 2. The remaining 6 stars (SW Scl, RW Sgr, R Pic, X Mon, $\mathrm{X}$ Oct, $\mathrm{V}$ CVn, $\mathrm{S}$ Acl) from this group are classified as Semiregulars in the GCVS-4 and were not included in our

\footnotetext{
${ }^{1}$ Note the different sign convention by Feast \& Whitelock (2000) for the $u$ component of space velocities. To avoid misunderstandings in the following discussion, we rename $u, v, w$ in Feast \& Whitelock (2000) as $U_{\mathrm{FW}}=-u, V_{\mathrm{FW}}=v, W_{\mathrm{FW}}=w$.
} 
Table 5. Mean kinematic and orbital characteristics of Miras.

\begin{tabular}{|c|c|c|c|c|c|c|c|c|c|c|}
\hline Type & $M$ & $M$ & $M$ & $M$ & $M$ & $M$ & $M$ & $M$ & $C$ & $S$ \\
\hline Period range & $<165$ & $165-225$ & $225-270$ & $270-315$ & $315-350$ & $350-385$ & $385-435$ & $>435$ & & \\
\hline No. of stars & 55 & 99 & 112 & 109 & 98 & 39 & 45 & 23 & 45 & 30 \\
\hline \multirow[t]{2}{*}{$\bar{U}\left[\mathrm{~km} \mathrm{~s}^{-1}\right]$} & +10.1 & -12.0 & +2.0 & -3.7 & -3.1 & -7.5 & +4.1 & +1.1 & -1.6 & -11.9 \\
\hline & \pm 11.4 & \pm 7.7 & \pm 7.2 & \pm 6.5 & \pm 5.2 & \pm 6.9 & \pm 7.8 & \pm 7.8 & \pm 7.8 & \pm 5.3 \\
\hline \multirow[t]{2}{*}{$\bar{V}\left[\mathrm{~km} \mathrm{~s}^{-1}\right]$} & -90.8 & -60.5 & -46.8 & -30.0 & -28.0 & -26.0 & -18.4 & -8.0 & -17.9 & -7.5 \\
\hline & \pm 18.9 & \pm 8.2 & \pm 6.4 & \pm 5.2 & \pm 4.1 & \pm 5.5 & \pm 4.9 & \pm 3.7 & \pm 5.7 & \pm 2.9 \\
\hline \multirow[t]{2}{*}{$\bar{W}\left[\mathrm{~km} \mathrm{~s}^{-1}\right]$} & -36.9 & -15.4 & -15.9 & -10.0 & -6.8 & -6.4 & +0.8 & -9.4 & -7.4 & -6.6 \\
\hline & \pm 9.5 & \pm 6.9 & \pm 6.1 & \pm 4.6 & \pm 3.7 & \pm 7.5 & \pm 2.3 & \pm 4.2 & \pm 6.7 & \pm 3.4 \\
\hline \multirow[t]{2}{*}{$\overline{\sigma_{U}}\left[\mathrm{~km} \mathrm{~s}^{-1}\right]$} & 81.5 & 76.3 & 76.2 & 68.0 & 50.8 & 42.3 & 35.5 & 36.4 & 55.0 & 28.5 \\
\hline & \pm 7.8 & \pm 5.5 & \pm 5.1 & \pm 4.6 & \pm 3.6 & \pm 4.8 & \pm 3.8 & \pm 5.5 & \pm 5.5 & \pm 3.7 \\
\hline \multirow[t]{2}{*}{$\overline{\sigma_{V}}\left[\mathrm{~km} \mathrm{~s}^{-1}\right]$} & 139.1 & 81.0 & 67.6 & 54.4 & 40.1 & 34.0 & 32.5 & 17.6 & 37.9 & 15.5 \\
\hline & \pm 13.4 & \pm 5.8 & \pm 4.5 & \pm 3.7 & \pm 2.9 & \pm 3.9 & \pm 3.5 & \pm 2.6 & \pm 4.0 & \pm 2.0 \\
\hline \multirow[t]{2}{*}{$\overline{\sigma_{W}}\left[\mathrm{~km} \mathrm{~s}^{-1}\right]$} & 70.2 & 68.3 & 64.6 & 48.1 & 36.7 & 46.4 & 15.2 & 19.5 & 44.6 & 18.4 \\
\hline & \pm 6.8 & \pm 4.9 & \pm 4.3 & \pm 3.3 & \pm 2.6 & \pm 5.3 & \pm 1.6 & \pm 2.9 & \pm 4.8 & \pm 2.4 \\
\hline \multirow[t]{2}{*}{$\overline{R_{\mathrm{p}}}[\mathrm{kpc}]$} & 4.98 & 4.60 & 4.76 & 5.21 & 5.47 & 5.61 & 5.66 & 6.16 & 6.00 & 6.32 \\
\hline & \pm 0.25 & \pm 0.18 & \pm 0.17 & \pm 0.17 & \pm 0.15 & \pm 0.22 & \pm 0.19 & \pm 0.16 & \pm 0.23 & \pm 0.18 \\
\hline \multirow[t]{2}{*}{$\overline{R_{\mathrm{a}}}[\mathrm{kpc}]$} & 9.91 & 9.12 & 9.13 & 9.31 & 8.97 & 8.75 & 8.84 & 8.90 & 9.58 & 8.83 \\
\hline & \pm 0.52 & \pm 0.26 & \pm 0.25 & \pm 0.19 & \pm 0.11 & \pm 0.10 & \pm 0.11 & \pm 0.13 & \pm 0.24 & \pm 0.12 \\
\hline \multirow[t]{2}{*}{$\overline{Z_{\max }}[\mathrm{kpc}]$} & 3.31 & 2.86 & 2.50 & 2.04 & 1.61 & 1.46 & 0.75 & 0.68 & 1.45 & 0.77 \\
\hline & \pm 0.42 & \pm 0.19 & \pm 0.18 & \pm 0.15 & \pm 0.10 & \pm 0.18 & \pm 0.08 & \pm 0.11 & \pm 0.20 & \pm 0.08 \\
\hline \multirow[t]{2}{*}{$\bar{e}$} & 0.321 & 0.332 & 0.319 & 0.293 & 0.252 & 0.230 & 0.228 & 0.183 & 0.235 & 0.169 \\
\hline & \pm 0.026 & \pm 0.017 & \pm 0.016 & \pm 0.017 & \pm 0.015 & \pm 0.020 & \pm 0.017 & \pm 0.016 & \pm 0.022 & \pm 0.012 \\
\hline
\end{tabular}

study. For the 11 stars in common, the individual values of the velocity components $U_{\mathrm{FW}}, V_{\mathrm{FW}}, W_{\mathrm{FW}}$ given in Feast \& Whitelock are consistent with our data $U, V, W$ (see Table 6). The mean difference in the $U$ component is $\overline{U-U_{\mathrm{FW}}}=-23 \pm 13 \mathrm{~km} \mathrm{~s}^{-1}$ (with the median value for the ratio $U_{\mathrm{FW}} / U$ being 1.2 ). Also the total space velocities in Feast \& Whitelock (2000) are, on average, by a factor of 1.2 higher then those derived in the present paper. Most probably, this effect is a consequence of different distance estimations (underestimated distances in the present paper, or overestimated in Feast \& Whitelock 2000, or both). Simple calculations support this explanation. Let us assume that, on average, a relation between the transverse $V_{\mathrm{tr}}$ and radial $V_{\text {rad }}$ velocity components is ${\overline{V_{\mathrm{tr}}}}^{2}=2{\overline{V_{\mathrm{rad}}}}^{2}$. Then the given difference in space velocities could arise due to distance estimations differing by a factor 1.4. If a PLR was used to derive the distances, an effect of this order would be caused by a difference in the estimation of absolute magnitudes of $\approx 0.7 \mathrm{mag}$. The data in Fig. 9 suggest this explanation. The direct comparison with the distances given in Whitelock et al. (2000) and those used by Feast \& Whitelock (2000) to compute the spatial velocities for these stars confirms the effect of the same order (which yields, finally, the difference by a factor of about 1.2 for the $U$ component).

Nevertheless, we conclude from Table 6 that 11 common stars with periods between 145 and 200 days and at distances less than $2 \mathrm{kpc}$ from the Sun show a strong
Table 6. Mean velocity components of Miras with periods in the range $145-200$ days $\left(U_{\mathrm{FW}}, V_{\mathrm{FW}}, W_{\mathrm{FW}}\right.$ from Feast \& Whitelock (2000); $U, V, W$ from the present study). Units: $\mathrm{km} \mathrm{s}^{-1}$

\begin{tabular}{lcccc}
\hline Feast \& & number & & & \\
Whitelock & of stars & $U_{\mathrm{FW}}$ & $V_{\mathrm{FW}}$ & $W_{\mathrm{FW}}$ \\
group 2 & 17 & $73 \pm 17$ & $-97 \pm 26$ & $-11 \pm 11$ \\
common stars & 11 & $75 \pm 22$ & $-109 \pm 26$ & $-17 \pm 16$ \\
\hline \hline & number & & & \\
this paper & of stars & $U$ & $V$ & $W$ \\
common stars & 11 & $52 \pm 18$ & $-97 \pm 23$ & $-23 \pm 13$ \\
$R<2$ kpc & 35 & $20 \pm 15$ & $-80 \pm 17$ & $-14 \pm 9$ \\
$R>2$ kpc & 42 & $-8 \pm 13$ & $-73 \pm 19$ & $-39 \pm 14$ \\
all Miras & 77 & $5 \pm 10$ & $-76 \pm 13$ & $-28 \pm 9$ \\
\hline
\end{tabular}

motion outwards from the Galactic Center. On the other hand, we must also stress that the subset considered by Feast \& Whitelock (2000) includes only a small portion of Miras known in the Solar neighborhood. Considering all 35 Miras identified within $2 \mathrm{kpc}$ from the Sun and assuming the possibility that the distances may be underestimated in our study, we cannot completely exclude the hypothesis of a net radial motion from the Galactic Centre for these stars, although, the effect should be of a considerably less significance. However, on the basis of all 
77 known Miras with periods in the range 145-200 days, we must reject the finding of an outward motion. Note also that the kinematic parameters derived for Miras in the near $(R<2 \mathrm{kpc})$ and far $(R>2 \mathrm{kpc})$ Solar neighborhood (see Table 6 ) do not justify a statistically significant separation into two different groups. Therefore, it is likely that the group 2 stars from Feast \& Whitelock (2000), which were selected on the basis of available Hipparcos proper motions, radial velocities and $K$ photometry, do not represent a general motion of Miras with periods in the range 145-200 days sufficiently well.

\subsection{Mira frequency}

In order to construct the luminosity function for the Miras, we computed absolute magnitudes $M_{V}$ for all 5042 stars with known periods by use of the PLR derived in this paper (see Fig. 6). A representative sample of Miras is accumulated in such a volume $Q$ where selection effects of observations and peculiarities of the spatial distribution in the galactic coordinate system $(X Y Z)$ can be compensated. As a compromise, we adopted a maximum distance from the Sun in the galactic plane $R=\left(X^{2}+Y^{2}\right)^{1 / 2}$ to be $0.5 \mathrm{kpc}$. Assuming an exponential decrease of the star density along the $Z$ coordinate, we defined the luminosity function as the number of Mira variables in the volume

$Q=\pi R^{2} \int_{-Z}^{Z} \exp \left(-z / h_{Z}\right) \mathrm{d} z=2 \pi R^{2} h_{Z}\left[1-\exp \left(-Z / h_{Z}\right)\right]$.

where the scale height $h_{Z}$ was taken from Kharchenko (1994) and $R=0.5 \mathrm{kpc}$. In order to decide which Miras are located within the volume $Q$, the distances were obtained from the solution of (13) and (14) in the same way as described above. Figure 10 shows the luminosity function of Mira variables in comparison with the function by Kharchenko (1994).

Furthermore, the luminosity function was used to derive the frequency functions $A_{\mathrm{c}}(V)$ and $A_{\mathrm{c}}(P)$ given in (1) and (2) and to predict the numbers of Miras to be expected from an all-sky survey complete up to a given limiting magnitude. The corresponding differential $A(V)$ and integrated $N(V)$ numbers of Mira variables were computed, assuming a distance of $8.5 \mathrm{kpc}$ of the Sun from the Galactic center. Figure 11 shows the predicted numbers of Miras per square degree in the directions to the Galactic center and anticenter.

From Figs. 1 and 11, we conclude that the proposed future astrometric space missions with a limiting magnitude of $V=15^{\mathrm{m}}$ (DIVA, FAME) or $V=20^{\mathrm{m}}$ (GAIA) will be able, in principle, to observe about 70000 or 135000 field Miras in the Galaxy, respectively. However, the number of Mira variables with accurate trigonometric parallaxes will be much less and will depend on the accuracy of the astrometric measurements. For example, DIVA (Röser 1999) will provide parallaxes at the 10\%-precision

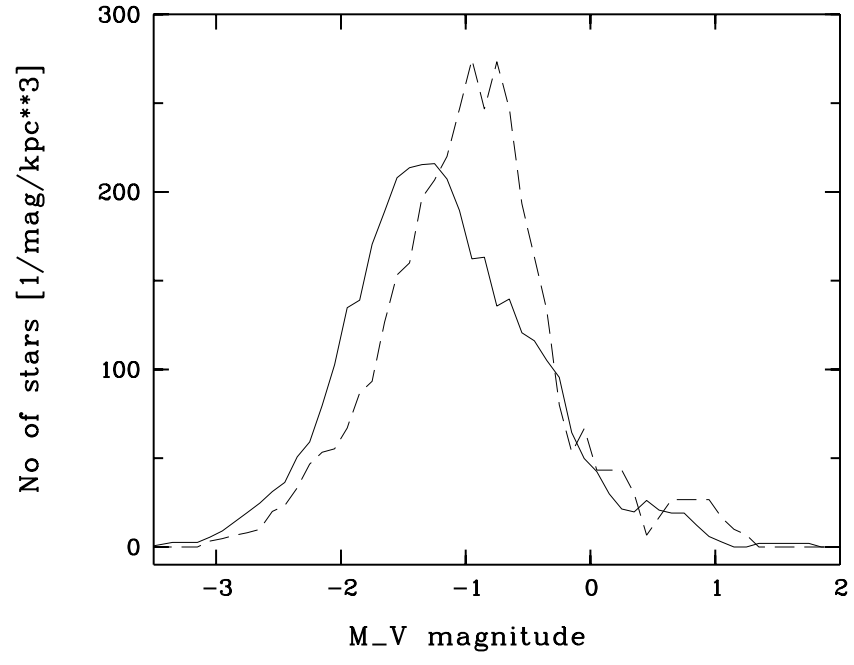

Fig. 10. The luminosity function of Mira variables based on the PLR which has been obtained in this paper (solid line) and by Kharchenko (1994) (dashed line).

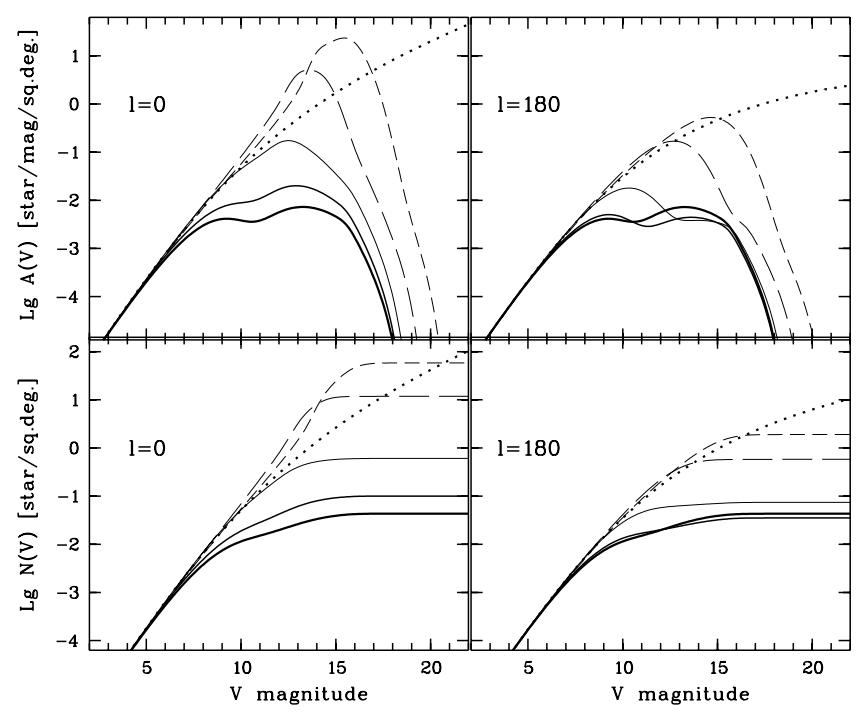

Fig. 11. The predicted differential $A(V)$ and integrated $N(V)$ numbers of Miras versus magnitude in the directions to the Galactic center $\left(l=0^{\circ}\right)$ and anticenter $\left(l=180^{\circ}\right)$. The dotted, short-dashed, long-dashed, thin, normal and thick lines mark the number of Miras at $b=0^{\circ}, 10^{\circ}, 20^{\circ}, 30^{\circ}, 60^{\circ}$ and $90^{\circ}$, respectively.

level for about 200 Miras. At present, the distance of not even a single Mira variable is known to this accuracy.

\section{Conclusions}

We took the information on Mira variables given in the GCVS-4 and selected a sample of stars for which new kinematic data were obtained during the last decade. Based on the statistical parallaxes, the mean absolute magnitudes and the spatial kinematic parameters were determined as functions of the pulsation periods. The BCs were derived for the oxygen-rich Miras and the PL relation was compared with predictions from pulsation theory and with other observations. The frequency of Mira variables was 
computed as a function of the visual magnitude. The following findings result from this analysis:

- With respect to Mira variables, the GCVS-4 is complete up to a magnitude at maximum of $V=9$.

- The method of statistical parallaxes provides a satisfactory distance scale.

- The BC-period relation of the oxygen-rich Miras shows a rather small dispersion and, at this stage, there is no evidence that it depends on the pulsation mode.

- The derived PLR $\left(M_{\mathrm{bol}}-\log P\right)$ is significantly steeper than the corresponding PLR found in the LMC (Feast et al. 1989) but in good agreement with the findings of Barthes et al. (1999) who studied galactic oxygenrich LPVs from the Hipparcos Catalogue. The derived shape of the PLR may be due to a significant dispersion of present masses and due to the existence of Mira populations of distinct ages in the solar neighbourhood.

- The comparison with theoretical pulsation models yields reasonable masses around $1 M_{\odot}$, consistent with previous studies. The most probable pulsation mode is the fundamental one.

- The kinematic parameters describing the spatial velocities and orbital motion indicate a higher age for the oxygen-rich Miras pulsating with smaller periods. That is in agreement with previous findings.

- The analyzed data do not support the assumption (Feast \& Whitelock 2000) of a significant motion radially outwards in the Galaxy for Miras in the period range 145-200 days.

Acknowledgements. We are grateful to Olga Durlevich for providing necessary data from the GCVS. N. Kharchenko and O. Malkov thank the Deutsche Forschungsgemeinschaft for financial support that enabled their visit to AIP Potsdam where this work has been done. E. Kilpio and O. Malkov also acknowledge support from the RF President grant 99-15-96022, and the Russian Academy of Sciences for the help provided in the frame of the "Young scientists support program". The authors also thank the anonymous referee for his/her very useful comments and suggestions.

\section{References}

Allen, C. W. 1973, Astrophysical quantities, 3d ed. (Athlone Press, London)

Alvarez, R., Mennessier, M. O., Barthès, D., Luri, X., \& Mattei, J. A. 1997, Hipparcos Venice'97, ESA SP-402, 383

Bahcall, J. N., \& Soneira, R. M. 1980, ApJS, 44, 73

Barbier-Brossat, M., \& Figon, P. 2000, A\&AS, 142, 217

Barnes, T. G., \& Fekel, F. C. 1977, The Observatory, 97, 1

Barthès, D. 1998, A\&A, 333, 647

Barthès, D., Luri, X., Alvarez, R., \& Mennessier, M. O. 1999, A\&AS, 140, 55

Barthès, D., \& Luri, X. 2001, A\&A, 365, 519

Bastian, U., \& Röser, S. 1993a, PPM Star Catalogue 3, 4 (Spektrum Academischer Verlag)

Bastian, U., \& Röser, S. 1993b, Veröffentlichungen Astronomisches Rechen Inst. (Heidelberg)

Bedding, T. R., \& Zijlstra, A. A. 1998, A\&A, 506, L47
Carlsberg Meridian Catalogue La Palma Numbers 1 to 11, Observations of positions of stars and planets: May 1984 to May 1998 (CMC11) 1999, Copenhagen University Observatory, Royal Greenwich Observatory and Real Instituto y Observatorio de la Armada en San Fernando, CD-ROM distribution

Clayton, M. L., \& Feast, M. V. 1969, MNRAS, 146, 411

Dean, C. A. 1976, AJ, 81, 364

Dehnen, W., \& Binney, J. J. 1998, MNRAS, 298, 387

ESA 1997, The Hipparcos and Tycho Catalogues, ESA SP-1200

Feast, M. W. 1963, MNRAS, 125, 367

Feast, M. W. 1966, MNRAS, 132, 495

Feast, M. 1999, in Asymptotic Giant Branch Stars, ed. T. Le Bertre, A. Lèbre, \& C. Waelkens, IAU Symp., 191, 109

Feast, M. W., Robertson, B. S. C., \& Black, C. 1980, MNRAS, 190, 227

Feast, M. W., \& Whitelock, P. 1997, MNRAS, 291, 683

Feast, M. W., \& Whitelock, P. 1999, in Post-Hipparcos Cosmic Candles, ed. A. Heck, \& F. Caputo (Kluwer), 75

Feast, M. W., \& Whitelock, P. 2000, MNRAS, 317, 460

Feast, M. W., Woolley, R., \& Yilmas, N. 1972, MNRAS, 158, 23

Høg, E., Kuzmin, A., Bastian, U., et al. 1998, A\&A, 335, L65

Høg, E., Fabricius, C., Makarov, V. V., et al. 2000, Astrometric and photometric reference catalogue of the 2.5 million brightest stars covering the entire sky, Copenhagen, CD-ROM distribution

Ikaunieks, J. J. 1971, Long period variable stars, Zinante, Riga Kharchenko, N. V. 1992, Kinemat. Phys. Celest. Bodies, 8, 61 Kharchenko, N. V. 1994, Kinemat. Phys. Celest. Bodies, 10, 70

Kholopov, P. N., Samus, N. N., Frolov, M. S., et al. 1985-1988, General Catalogue of Variable Stars, 4th edition (Nauka Publishing House, Moscow) (GCVS-4)

Kilpio, E. Yu., \& Malkov, O. Yu. 1997, Astron. Rep., 41, 10

Kukarkin, B. V., Kholopov, P. N., Efremov, Yu. N., et al. 1969, General Catalogue of Variable Stars, 3rd edition (Nauka Publishing House, Moscow) (GCVS-3)

Kukarkin, B. V., Kholopov, P. N., Artiukhina, N. M., et al. 1982, New Catalogue of Suspected Variable Stars (Nauka Publishing House, Moscow)

Osvald, V., \& Risley, A. M. 1961, Publ. Leander McCormick Obs., 11, 147

Percy, J. R., \& Colivas, T. 1999, PASP, 111, 94

Perry, C. L., \& Bidelman, W. P. 1965, PASP, 77, 214

Röser, S. 1999, in Reviews in Modern Astronomy 12, ed. R. E. Schielicke (Astronomische Gesellschaft, Hamburg), 97

Röser, S., \& Bastian, U. 1991, PPM Star Catalogue 1, 2 (Spektrum Academischer Verlag)

Saio, H., \& Yoshii, Y. 1979, PASP, 91, 553

Sharov, A. S. 1963, Astron. Zh., 40, 900

Smak, J. I., \& Preston, G. W. 1965, ApJ, 142, 943

Straižys, V. 1992, Multicolor stellar photometry (Pachart Publishing House, Tucson, Arizona)

Tuchman, Y. 1999, in Asymptotic Giant Branch Stars, ed. T. Le Bertre, A. Lèbre, \& C. Waelkens, IAU Symp., 191, 122

Urban, S. E., Corbin, T. E., \& Wycoff, G. L. 1998, AJ, 115, 2161

van Leeuwen, F., Feast, M. W., Whitelock, P. A., \& Yudin, B. 1997, MNRAS, 287, 955

Whitelock, P., \& Feast, M. 2000, MNRAS, 319, 759

Whitelock, P., Marang, F., \& Feast, M. 2000, MNRAS, 319, 728

Wood, P. R., \& Sebo, K. M. 1996, MNRAS, 282, 958

Ya'ari, A., \& Tuchman, Y. 1996, ApJ, 456, 350 